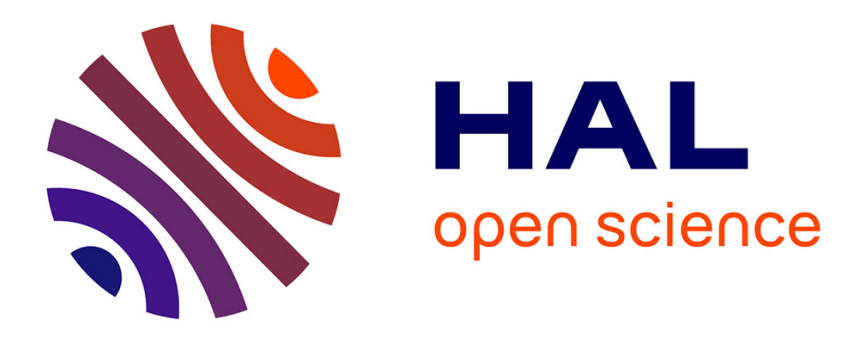

\title{
Stability intervals of metastable states in hysteretic systems \\ Joël Puibasset
}

\section{To cite this version:}

Joël Puibasset. Stability intervals of metastable states in hysteretic systems. Physical Review E: Statistical, Nonlinear, and Soft Matter Physics, 2011, 84 (6), 10.1103/PhysRevE.84.061126 . hal02110039

\section{HAL Id: hal-02110039 \\ https://hal.science/hal-02110039}

Submitted on 26 Nov 2019

HAL is a multi-disciplinary open access archive for the deposit and dissemination of scientific research documents, whether they are published or not. The documents may come from teaching and research institutions in France or abroad, or from public or private research centers.
L'archive ouverte pluridisciplinaire HAL, est destinée au dépôt et à la diffusion de documents scientifiques de niveau recherche, publiés ou non, émanant des établissements d'enseignement et de recherche français ou étrangers, des laboratoires publics ou privés. 


\title{
Stability Intervals of Metastable States in Hysteretic Systems
}

\author{
Joël Puibasset* \\ CRMD , CNRS-Université d'Orléans, $1 b$ rue de la Férollerie, 45071 Orléans Cedex 02, France.
}

(Dated: November 21, 2011)

\begin{abstract}
Hysteresis in disordered systems originates in a plethora of metastable states. Previous works focused on their distribution inside the hysteresis. In contrast, we propose an analysis of their range of metastability. Our model, designed to catch the main features of fluid adsorption in porous materials, shows strong evidences that, in the thermodynamic limit, despite metastable states of finite range can be found, they are exponentially dominated by those infinitely localized.

PACS numbers: 64.60.My, 68.43.De, 75.60.Ej, 05.10.Ln
\end{abstract}

\section{INTRODUCTION}

Magnetic systems are famous examples where the hysteresis originates in a plethora of metastable states associated to a free energy landscape [1]. It is also the case for adsorption of fluid in nanoporous materials, as shown by recent studies $[2-5]$. In these systems, the metastable states correspond to fluid configurations in the porous network [6-8], which can be seen as a collection of interconnected domains [9-11] associated to nano-cavities and/or physico-chemical heterogeneities.

These metastable states may be (partially) explored while measuring the adsorption/desorption isotherms, which give the amount of fluid adsorbed as a function of the chemical potential $\mu$, as used in standard method of porous materials characterization [12]. Understanding their distribution and properties inside the hysteresis would be extremely helpful to experimental data analysis, besides being a formidable challenge to understand physics of complex disordered systems [13-17].

A strong connection has been shown between the region where the density of metastable states grows exponentially with the system size, the hysteresis, and the possible occurrence of an underlying macroscopic transition with reentrance $[5,18,19]$. However, the range of existence of the metastable states has attracted limited attention. In finite size systems, a given metastable state exists on an interval defined by the lower and higher metastability limits $\left[\mu_{-}, \mu_{+}\right]$. How does the distribution of metastability ranges $\Delta \mu=\mu_{+}-\mu_{-}$look like, and what do we expect in the thermodynamic limit? Such issues have not been addressed so far. In this paper, we answer these questions in the framework of a molecular model of fluid adsorbed in heterogeneous linear nanopores.

\section{MODEL AND METHODS}

Our model, introduced in a previous work on hysteresis and scanning curves [20,21], incorporates the main phys-

*Electronic address: puibasset@cnrs-orleans.fr ical ingredients characterizing fluid adsorption in porous materials:

1) Linear nanopores are representative of a large class of real materials like MCM-41 [22, 23], porous silicon [24][32], or nanoporous alumina, possibly modulated at mesoscopic scale [25-27].

2) These materials generally exhibit large scale (several nanometers) physico-chemical heterogeneities. The pore can be seen as a string of more or less attractive domains. Taking into account the interdependence between these domains is made easy in this one-dimensional topology.

3) The molecular description provides a realistic treatment and a clear physical interpretation of the metastable states. All calculations are based on interatomic potentials, without need for the introduction of ad-hoc rules as in usual pore network models which may miss some important features.

The nanopore (diameter: $3 \mathrm{~nm}$ ) is drilled in pure silica, a widely used material. The adsorbed fluid is Argon since it is commonly used as a simple fluid in many experiments. All interactions are supposed to follow the Lennard-Jones $(12,6)$ potential truncated at 2.5 atomic diameters for convenience. The parameters are those for argon-argon and argon-oxygen species to describe fluidfluid and fluid-silica interactions [20].

Besides the spontaneous atomic roughness, one introduces heterogeneities at nanometric scale as follows. The nanopore is divided into $7 \mathrm{~nm}$ long domains. For each domain, a random magnification factor $h$ is applied to the fluid/wall interaction parameter, according to a Gaussian distribution centered on 1 and variance 0.04 (weak modulation). The system thus appears as a string of domains with random affinity for the fluid. The interdependence between these domains is taken into account. The results will thus depend on the pore sample characterized by a set of $h$-values, a so-called "disorder realization". Appropriate average over disorder will be introduced to produce sensible results.

For each domain and each $h$-value, the numerical calculations are performed in the standard Grand Canonical Monte Carlo (GCMC) framework, giving the amount of fluid adsorbed as a function of $\mu$, except that the usual periodic boundary conditions along the pore axis are modified to take into account the fluid state in the other domains. Since interactions and correlations are short 


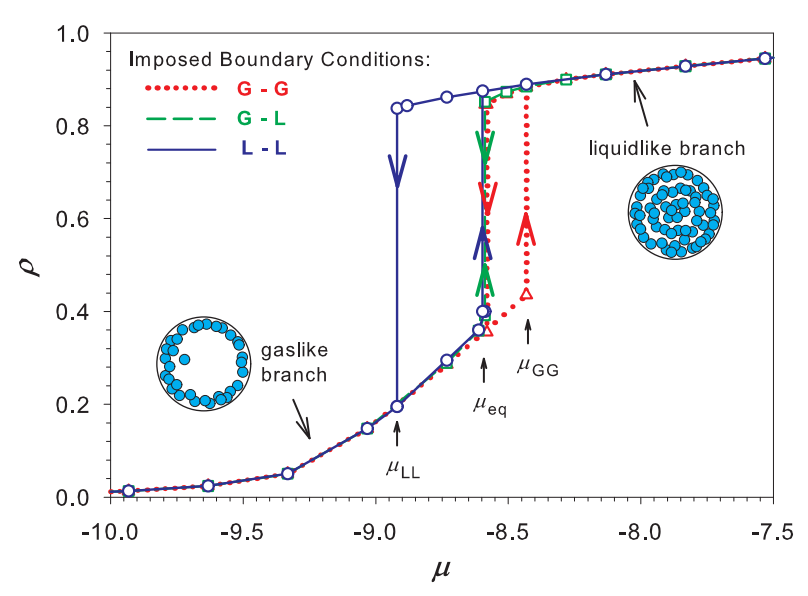

FIG. 1: (color online). Symbols: conditional isotherms obtained by Grand Canonical Monte Carlo with imposed boundary conditions. The results are given for a domain with $h=1.0$, and the uncertainties are less than symbol sizes. Lines are guide to the eye. The domain is either filled by liquidlike argon (liquidlike branch, L) or by gas with argon adsorbed at the walls (gaslike branch, G). The G-L conditional isotherm is reversible (transition occurs at $\mu_{\mathrm{eq}}$ ), while the G-G and L-L conditional isotherms exhibit large hysteresis (the transitions occur at $\mu_{\mathrm{GG}}$ and $\mu_{\mathrm{eq}}$ for $\mathrm{G}-\mathrm{G}$, and $\mu_{\mathrm{eq}}$ and $\mu_{\mathrm{LL}}$ for $\left.\mathrm{L}-\mathrm{L}\right)$.

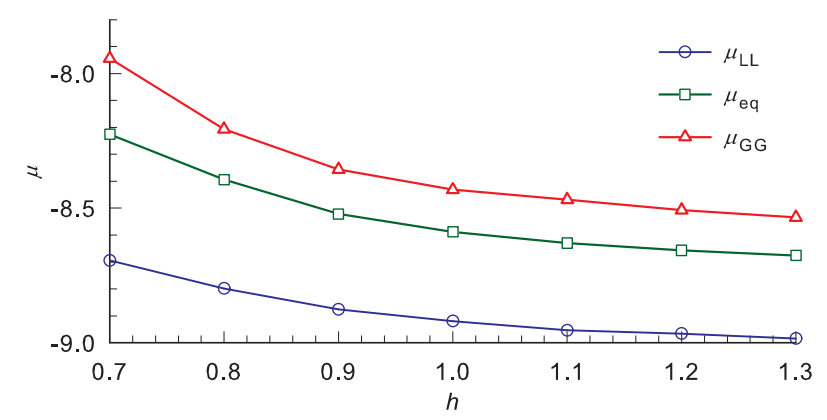

FIG. 2: (color online). Evolution of the three chemical potentials where transitions occur in the conditional isotherms (see Fig. 1), as a function of the heterogeneity parameter $h$.

ranged, the nearest neighboring domains alone need to be considered. The details in the neighboring domains are actually irrelevant, and it is enough to know the average fluid density.

The GCMC algorithm produces a set of molecular configurations representative of the grand canonical ensemble. The algorithm generates a random walk in phase space by randomly moving the particles. The acceptance rules given by the Metropolis algorithm then select the molecular configurations according to their statistical importance. Statistical averages are then performed on these molecular configurations to get all thermodynamic properties, in particular the conditional isotherms.

The Monte Carlo results are given in Fig. 1 for $h=1.0$.
As can be seen, the domain behaves essentially as a bistable system. The fluid may either follow the gaslike branch, denoted G, corresponding to the fluid adsorbed only at the walls, or follow the liquidlike branch, denoted L, corresponding to the fluid filling the whole domain. The condensation occurs either at equilibrium $\left(\mu_{\text {eq }}\right)$ if at least one neighbor is liquidlike, or is delayed if both neighbor are gaslike ( $\mu_{\mathrm{GG}}$ corresponds to liquid nucleation). The domain emptying occurs at $\mu_{\text {eq }}$ if at least one neighbor is gaslike, or is delayed if both neighbors are liquidlike ( $\mu_{\mathrm{LL}}$ corresponds to cavitation). The domain is thus reversible if one neighbor is gaslike and the second one is liquidlike. Conversely, the domain exhibits distinctive hysteretic behavior if both domains are both either gaslike or liquidlike. Three "conditional isotherms" are thus obtained, as shown in Fig. 1.

The reversibility of the transition in a domain when one neighbor is gaslike and the other is liquidlike originates in the disappearance of the nucleation barrier. During adsorption, the liquidlike neighboring domain provides the required liquid nucleus for capillary condensation, while during desorption the gaslike neighboring domain provides the bubble nucleus necessary to domain emptying. The transition thus proceeds reversibly via meniscus advancing (filling) or receding (emptying) in the domain.

Extensive calculations show that the conditional isotherms depend only on the $h$-value in the considered domain, whatever the $h$-values in the neighboring domains. This greatly simplifies the problem. In practice, the molecular simulations are performed for a discrete set of $h \in[0.7,0.8,0.9,1.0,1.1,1.2,1.3]$ and then interpolated for any $h$-value. Magnification factors less than 0.7 or larger than 1.3 are discarded (to be discussed in last section). Fig. 2 gives the variations with $h$ of the three chemical potential values characterizing the conditional isotherms $\mu_{\mathrm{LL}}, \mu_{\mathrm{eq}}$ and $\mu_{\mathrm{GG}}$.

Once these conditional isotherms are known, simple algorithms may be used to determine the fluid behavior in any given pore sample (adsorption/desorption isotherms, scanning curves, metastable states). Starting from any given initial fluid configuration, including the empty or saturated states, small positive or negative $\mu$-increments may be applied to the system. This change in $\mu$ impacts on the amount of fluid adsorbed in all domains, as given by the conditional isotherms. For most of them, the variation is continuous, but a sharp transition may occur in few domains. This affects the fluid status in these domains, and consequently the conditional isotherms in the neighboring domains. It may trigger subsequent transitions (avalanche), until a new metastable state is reached.

$N$ domains in the pore lead to at most $2^{N}$ possible fluid states. Few of them are consistent with the conditional isotherms. They are stable against the thermal fluctuations, taken into account in the pre-calculated isotherms: they are metastable. 


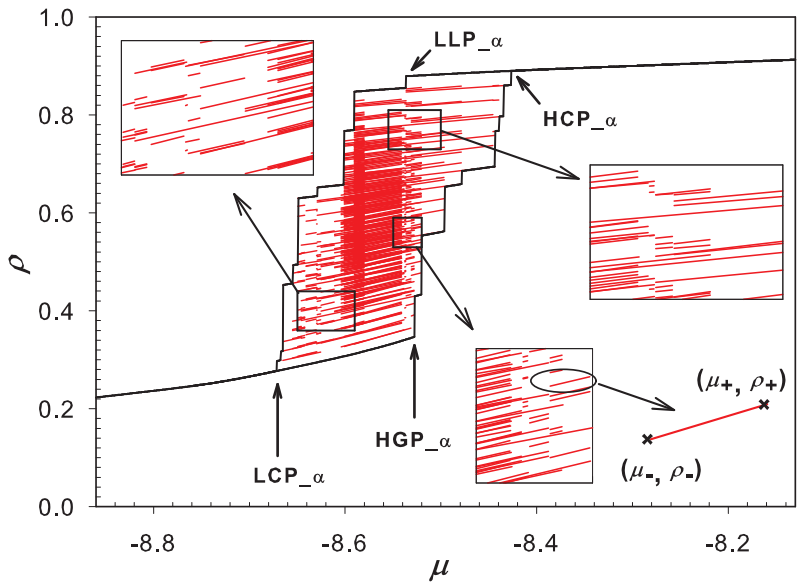

FIG. 3: (color online). Main adsorption/desorption hysteresis (continuous line) and metastable states (portions of lines inside the hysteresis loop) for a particular disorder realization (denoted by $\alpha$ ) of a pore with 25 domains. $\mu_{-}$and $\mu_{+}$are the lower and higher metastability limits for a given metastable state. The metastable states are essentially parallel; the main hysteresis is the envelope of the metastable states; and the system exhibits a large variety of metastability ranges $\Delta \mu=\mu_{+}-\mu_{-}$everywhere in the loop (magnifications). These features hold for any disorder realization.

\section{NUMERICAL RESULTS}

For small systems $(N \leq 30)$, a systematic enumeration of all metastable states is possible. An example is given in Fig. 3. It is a remarkable feature that metastable states with a large variety of stability ranges $\Delta \mu$ can be found everywhere in the hysteresis. Let us introduce $\mathcal{N}_{N}^{\alpha}\left(\mu_{-}, \mu_{+}\right)$the density of metastable states with metastability limits $\mu_{-}$and $\mu_{+}$, where $\alpha$ denotes a particular disorder realization. For numerical evaluations, all chemical potentials are discrete, with a mesh $\delta \mu=3 \times 10^{-3}$ chosen to be sufficiently small not to affect the results.

The density $\mathcal{N}_{N}^{\alpha}\left(\mu_{-}, \mu_{+}\right)$is expected to grow exponentially with system size. One thus introduces its normalized logarithm

$$
\Sigma_{N}^{\alpha}\left(\mu_{-}, \mu_{+}\right)=\frac{1}{N} \ln \mathcal{N}_{N}^{\alpha}\left(\mu_{-}, \mu_{+}\right) .
$$

$\lim _{N \rightarrow \infty} \Sigma_{N}^{\alpha}\left(\mu_{-}, \mu_{+}\right)$is expected to converge. Fig. 4 shows that $\Sigma_{N}^{\alpha}\left(\mu_{-}, \mu_{+}\right)$may be positive anywhere in the physical region imposed by the closure points of the hysteresis, except for $\Delta \mu>0.2$. This confirms the previous qualitative observation that metastable states of various range of stability can be found everywhere in the hysteresis. However, for a given $\mu$, the largest values of $\Sigma_{N}^{\alpha}\left(\mu_{-}, \mu_{+}\right)$are found for small $\Delta \mu$. One may then suspect that, in the thermodynamic limit $N \rightarrow \infty$, localized metastable states $(\Delta \mu \rightarrow 0)$ are exponentially favored. To address this question, one has to somehow perform an average over disorder and a system size analysis.

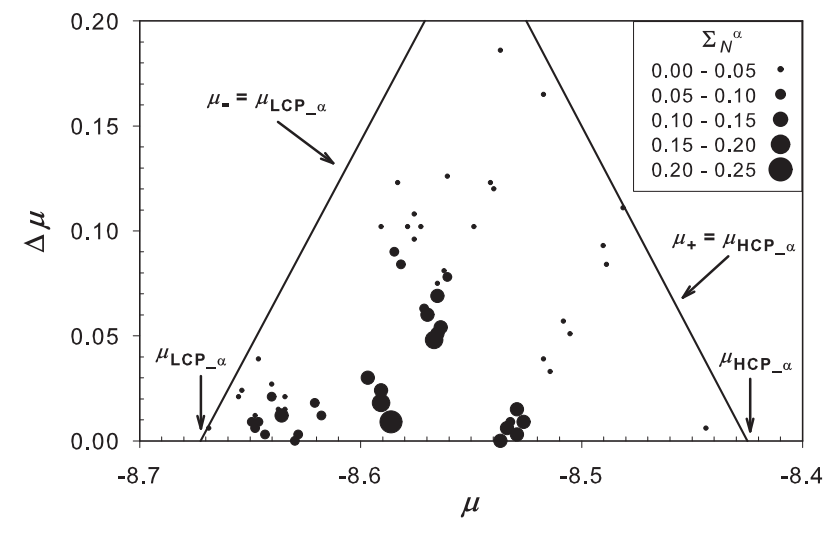

FIG. 4: Normalized logarithm of the number of metastable states $\Sigma_{N}^{\alpha}$ as a function of $\mu=0.5\left(\mu_{-}+\mu_{+}\right)$and $\Delta \mu=\mu_{+}-\mu_{-}$ for the same system as in Fig. 3. The amplitude is given by disc size. The solid lines materialize the upper physical limit for $\Delta \mu$ imposed by the lower and higher closure points of the hysteresis (LCP_ $\alpha$ and HCP_ $\alpha$ in Fig. 3). Discrete values result from the poor statistics associated to a single pore realization, to be smeared out by average over disorder.

In the thermodynamic limit, large subsystems are essentially independent. Assuming this is true for any subsystem size gives a general procedure to perform averages in disordered systems. Applying this to the density of metastable states in the hysteresis leads to the correct quenched complexity $[13,28]$. It is now applied to $\mathcal{N}_{N}^{\alpha}\left(\mu_{-}, \mu_{+}\right)$.

Let us consider two (independent) subsystems $\alpha$ and $\beta$, and their reunion $\alpha \cup \beta$. Chemical equilibrium imposes identical $\mu$ in both subsystems. As a consequence, for a given $\mu$, there is a one to one correspondence between the metastable states of $\alpha \cup \beta$, and the combinations of $\alpha$-metastable states and $\beta$-metastable states. This simple correspondence fails if one wants to consider the metastable states and their interval of metastability. For instance, the intervals of metastability of the $\alpha$ and $\beta$ states may be different and larger than the one for the $\alpha \cup \beta$ state. More precisely, chemical equilibrium imposes that

$$
\left[\mu_{-}^{\alpha \cup \beta}, \mu_{+}^{\alpha \cup \beta}\right]=\left[\mu_{-}^{\alpha}, \mu_{+}^{\alpha}\right] \cap\left[\mu_{-}^{\beta}, \mu_{+}^{\beta}\right] .
$$

The one to one correspondence depicted above may be recovered if one takes into account all states with a range of metastability including a given interval $\left[\mu_{-}, \mu_{+}\right]$: any such metastable state for $\alpha \cup \beta$ is made of an $\alpha$-state and a $\beta$-state which are metastable on intervals including $\left[\mu_{-}, \mu_{+}\right]$, and conversely. One thus introduces the number of states with a range of metastability including a given interval $\left[\mu_{-}, \mu_{+}\right]$:

$$
\mathcal{Q}_{N}^{\alpha}\left(\mu_{-}, \mu_{+}\right)=\int_{-\infty}^{\mu_{-}} \int_{\mu_{+}}^{+\infty} \mathcal{N}_{N}^{\alpha}\left(\mu_{-}^{\prime}, \mu_{+}^{\prime}\right) d \mu_{-}^{\prime} d \mu_{+}^{\prime}
$$

This quantity is multiplicative:

$$
\mathcal{Q}_{N}^{\alpha \cup \beta}\left(\mu_{-}, \mu_{+}\right)=\mathcal{Q}_{N}^{\alpha}\left(\mu_{-}, \mu_{+}\right) \times \mathcal{Q}_{N}^{\beta}\left(\mu_{-}, \mu_{+}\right) .
$$




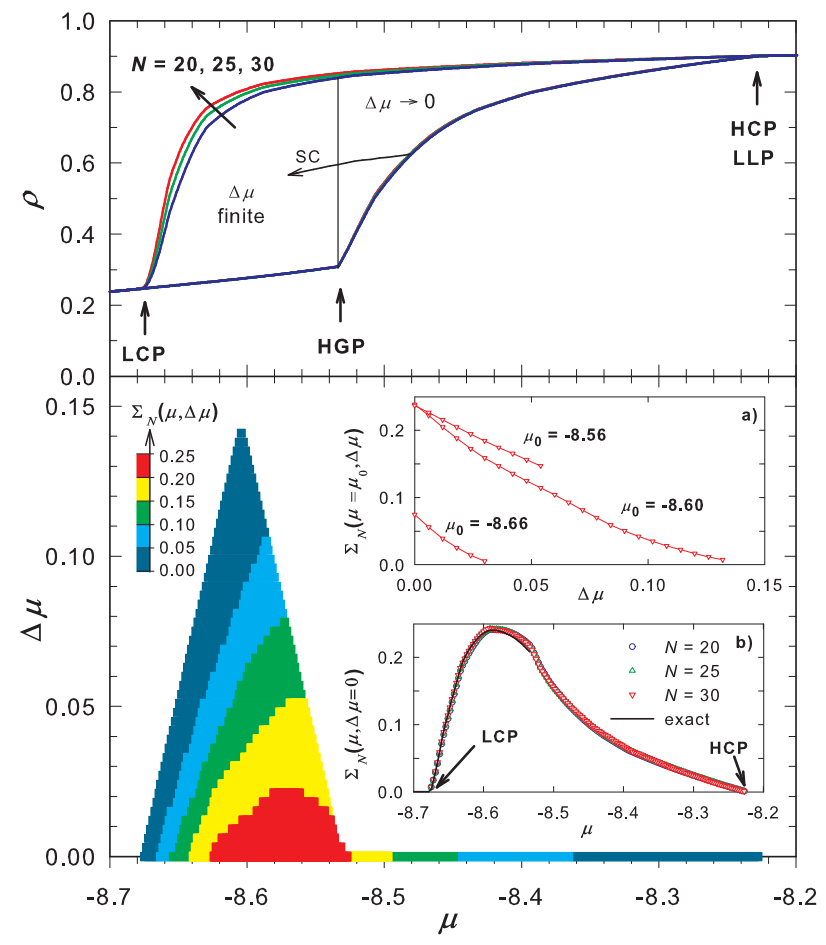

FIG. 5: (color online). Lower panel: Normalized logarithm of the number of metastable states with range of metastability $\left[\mu_{-}, \mu_{+}\right]$properly averaged over disorder, $\Sigma_{N}\left(\mu_{-}, \mu_{+}\right)$, for $N=30$, given as a function of $\mu=0.5\left(\mu_{-}+\mu_{+}\right)$and $\Delta \mu=\mu_{+}-\mu_{-}$. Inset a: $\Sigma_{N}(\mu, \Delta \mu)$ for three $\mu$-values. Inset b: symbols: $\Sigma_{N}(\mu, \Delta \mu=0)$ for various $N$; solid line: analytical determination in the thermodynamic limit for $\mu<-8.53$. Upper panel: average adsorption/desorption isotherm for various system sizes. Thin line: partial descending scanning curve (SC).

The appendix proposes a different method leading to the same result. Eq. 4 shows that the normalized logarithm of $\mathcal{Q}_{N}^{\alpha \cup \beta}\left(\mu_{-}, \mu_{+}\right)$is self-averaging [33]. Performing the average leads to the quantity

$$
\mathcal{Y}_{N}\left(\mu_{-}, \mu_{+}\right)=\frac{1}{N} \overline{\ln \mathcal{Q}_{N}^{\alpha}\left(\mu_{-}, \mu_{+}\right)}
$$

where the bar denotes the average over disorder. $\mathcal{Y}_{N}\left(\mu_{-}, \mu_{+}\right)$is expected to have negligible fluctuations in the thermodynamic limit (scaling as $1 / \sqrt{N}$ ).

The properly averaged density of metastable states is recovered by double differentiation (inversion of Eq. 3):

$$
\mathcal{N}_{N}\left(\mu_{-}, \mu_{+}\right)=-\frac{\partial^{2}}{\partial \mu_{-} \partial \mu_{+}} e^{N \mathcal{Y}_{N}\left(\mu_{-}, \mu_{+}\right)} \geq 0
$$

When $\mathcal{Y}_{N}\left(\mu_{-}, \mu_{+}\right)$is locally flat, $\mathcal{N}_{N}\left(\mu_{-}, \mu_{+}\right)=0$. It is otherwise an exponentially large number, whose corresponding entropy is $\Sigma_{N}\left(\mu_{-}, \mu_{+}\right)=\frac{1}{N} \ln \mathcal{N}_{N}\left(\mu_{-}, \mu_{+}\right)$. Its leading term is $\mathcal{Y}_{N}\left(\mu_{-}, \mu_{+}\right)$.

$\Sigma_{N}\left(\mu_{-}, \mu_{+}\right)$is shown in Fig. 5 (color map in the lower panel) for $N=30$ (5000 disorder realizations) as a func- tion of $\mu$ and $\Delta \mu$. Note that data are now smooth (compare with Fig. 4). Two regions where $\Sigma_{N}\left(\mu_{-}, \mu_{+}\right)>0$ clearly appear: a triangular shape and a line around $\Delta \mu=0$. The latter corresponds to metastable states with an arbitrarily small range of stability (in practice smaller than our discrete parameter $\left.\delta \mu=3 \times 10^{-3}\right)$. The corresponding curve $\Sigma_{N}(\mu, \Delta \mu=0)$ is given in inset b (down triangles). $\Sigma_{N}(\mu, \Delta \mu=0)$ is positive only for $\mu$ within the hysteresis region defined by the lower and higher closure points of the average isotherm (LCP and HCP, see Fig. 5, higher panel), and exhibit a single maximum in the core of the hysteresis. The results, essentially independent of system size (inset b), suggest that the thermodynamic limit has been reached. This is confirmed by the exact (analytical) determination of $\lim _{N \rightarrow \infty} \Sigma_{N}(\mu, \Delta \mu=0)$ for $\mu<-8.53$ (solid line in inset b; the calculation follows Appendix B in Ref. 5). This leads to the conclusion that our heterogeneous linear pore typically exhibits an exponentially large number of metastable states with a negligible range of metastability for all $\mu$-values in the hysteresis.

Fig. 5 reveals the existence of metastable states of finite $\Delta \mu$. The left boundary of the triangle corresponds to $\mu_{-}=\mu_{\mathrm{LCP}}$. The right boundary corresponds to $\mu_{+}=$ $\mu_{\mathrm{HGP}}$. This point is the high stability limit of the lowest (gaslike) metastable state. It corresponds to the first nucleation of a liquidlike droplet, to occur in the most attractive domain of the pore (lowest value of $\mu_{\mathrm{GG}}$ given in Fig. 2). This value splits the hysteresis in two regions:

1) Below $\mu_{\mathrm{HGP}}, \Sigma_{N}(\mu, \Delta \mu)$ is a smoothly decreasing function of $\Delta \mu$ as depicted in Fig. 5 (inset a) for three values of $\mu$. Therefore, metastable states of finite range can be found. But, in the thermodynamic limit, metastable states of smallest range $(\Delta \mu \rightarrow 0)$ exponentially dominate the others. Note the smooth variation of $\Sigma_{N}(\mu, \Delta \mu)$ around $\Delta \mu=0$ : the metastable states of negligible range of stability thus appear as a limiting case of metastable states of finite range.

2) Above $\mu_{\mathrm{HGP}}$, metastable states of finite range $(\Delta \mu>\delta \mu)$ are totally suppressed by average over disorder. But an exponentially large number of metastable states of negligible range can still be found.

The interpretation of these results is not straightforward. Qualitative arguments can however be given regarding the metastable states close to the gaslike and liquidlike branches. The region defined by the acceptable values in Fig. 5 is reminiscent of the triangular region depicted in Fig. 4 for one pore sample. Averaging over disorder actually selects a sub-region compatible for all pore realizations. The left boundary corresponds to metastable states with the lowest possible $\mu_{-}$, given by $\min _{\alpha} \mu_{\mathrm{LCP} \_} \alpha=-8.676=\mu_{\mathrm{LCP}}$. They are closely parallel to the gaslike branch. Those with the largest range of metastability end up at $\min _{\alpha} \mu_{\mathrm{HGP}_{\alpha} \alpha}=-8.534=\mu_{\mathrm{HGP}}$. The behavior close to the liquidlike branch is completely different. The lowest stability limit of the highest (liquidlike) metastable state $\mu_{\mathrm{LLP}}=\max _{\alpha} \mu_{\mathrm{LLP} \_\alpha}=-8.226$ reaches the higher closure point of the average hysteresis 


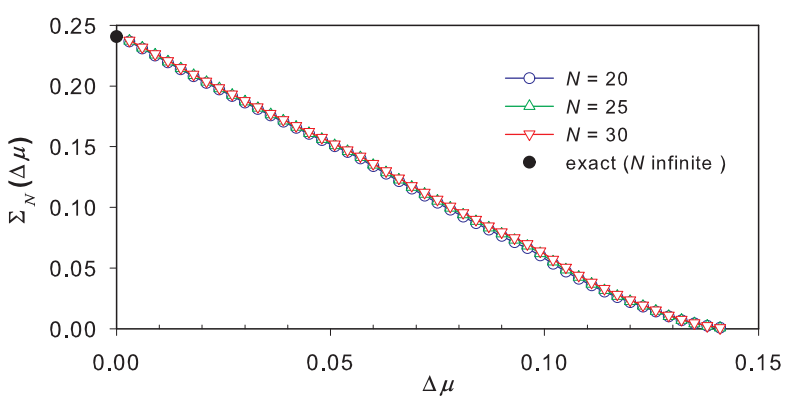

FIG. 6: (color online). Symbols: Normalized logarithm of the distribution of metastability ranges $\Delta \mu$ in the hysteresis $\Sigma_{N}(\Delta \mu)$, for various system sizes $N=20,25,30$. Closed circle: analytical determination of $\Sigma_{N \rightarrow \infty}(\Delta \mu=0)$.

$\mu_{\mathrm{LCP}}=-8.226$, and one cannot find metastable states of finite range close to the liquidlike branch.

The distribution of all metastability ranges in the hysteresis is obtained by integration of $\exp \left[N \Sigma_{N}(\mu, \Delta \mu)\right]$ with respect to $\mu$. Introducing the normalized logarithm, the leading term is given by the maximum of the exponent: $\Sigma_{N}(\Delta \mu)=\max _{\mu} \Sigma_{N}(\mu, \Delta \mu)$.

The results, given in Fig. 6, show a weak dependence with system size. Furthermore, they are in agreement with the exact value for $\Delta \mu=0$ and $N \rightarrow \infty$, suggesting they can be extrapolated to the thermodynamic limit. The smooth decrease of $\Sigma_{N}(\Delta \mu)$ supports the important conclusion that in heterogeneous linear nanopores, the metastable states of negligible range $(\Delta \mu=0)$ are exponentially dominant. There is however an exponentially large number of metastable states with a finite range of metastability, in close connection with the behavior of the lowest (gaslike) and highest (liquidlike) metastable states.

\section{DISCUSSION AND PERSPECTIVES}

As mentioned in "Model and Methods", magnification factors $h$ less than 0.7 or larger than 1.3 were discarded. What would change in the results if such domains were considered? Their direct contribution to fluid properties (amount adsorbed for instance) is negligible since they are exponentially rare. However, they can play an important role regarding nucleation (adsorption) and cavitation (desorption). For instance, the position of the right boundary of the triangular region in Fig. 5 is given by the first nucleation of a liquid droplet. It is given by $\mu_{\mathrm{HGP}}$, the lowest value of $\mu_{\mathrm{GG}}$ given in Fig. 2. Considering $h$-values larger than 1.3 would slightly reduce $\mu_{\mathrm{HGP}}$. Note however that $\mu_{\mathrm{GG}}$ saturates with $h$, and the qualitative behavior depicted in Fig. 5 thus remains unaffected. On the other hand, domains with low $h$-values are those with extremely low affinity for the fluid, which remain empty even at saturation. Their possible role as bubble nucleation centers upon desorption is irrelevant in open pores, where the first domain directly in contact with the reservoir already plays this role.

What is the connection between stability intervals of metastable states and observed hysteresis? Are metastable states of negligible range relevant? Of course, only accessible states, which can be reached by a $\mu$ history, are relevant to hysteresis. However, the connection between stability intervals of metastable states and their accessibility cannot be established rigorously because it is impossible to evaluate the accessibility of all metastable states. The shape of the main hysteresis loop seems to be governed solely by the metastable states with finite range. Furthermore, intuition suggests that infinitely localized states might be inaccessible. However, Fig. 5 reveals that the hysteresis also extends to the region where the density of metastable states with finite range is negligible (above $\mu_{\mathrm{HGP}}$ ). To explore the core of the hysteresis, a partial descending scanning curve is also shown (denoted SC in Fig. 5). There is no visible change in slope while crossing the vertical thin line, suggesting that the existence of a huge number of metastable states of finite range has a negligible influence on the scanning curve. These observations suggest at least two possibilities regarding the connection between the metastable states and the hysteresis: the relevant states are either the huge number of metastable states of negligible range, or a limited number of metastable states of finite range. Experimental observations may help to draw conclusions, to be discussed now.

How do these results compare to experiments? It is unfortunately impossible for experimentalists to perform direct measurements of ranges of metastability. However, two predictions of the model are relevant to experiments. 1) The metastable state of lowest density has a finite range which ends up at $\mu_{\mathrm{HGP}}>\mu_{\mathrm{LCP}}$. As a consequence, any descending scanning curve starting below $\mu_{\text {HGP }}$ reversibly follows the state of lowest density (adsorption branch), while descending scanning curves starting above $\mu_{\mathrm{HGP}}$ exhibit irreversibility. 2) If metastable states of finite range could be reached within the hysteresis, the system would exhibit local reversibility, which is not supported by experiments. It suggests that these states of finite range are probably not accessible, and the relevant accessible states would be the infinitely localized metastable states.

Further investigations will be necessary to address these issues. Future work will also explore the effects of temperature, dimensionality and range of interactions.

\section{Acknowledgments}

E. Kierlik and G. Tarjus are gratefully acknowledged for stimulating discussions. The simulations were performed thanks to the grant i2010091153 of the Institut de Développement des Ressources en Informatique Scientifique, IDRIS-CNRS, Orsay, France. 


\section{Appendix}

This appendix proposes another route to Eq. 4. We first focus on a slightly simplified problem where it is assumed that all metastable states have a fixed lower metastability limit $\mu_{-}=\mu_{0}$. We are thus interested in the distribution of their upper metastability limit $\mu_{+} \geq \mu_{0}$. The number of metastable states ending at $\mu_{+}$is now denoted $\mathcal{N}_{N}^{\alpha}\left(\mu_{+}\right)$, where $\mu_{-}$is temporarily dropped, and $\alpha$ denotes a particular disorder realization or a subsystem. Any combination of $\alpha$ and $\beta$ metastable states will produce a metastable state for the reunion $\alpha \cup \beta$ of the two subsystems. Its upper limit of stability $\mu_{+}^{\alpha \cup \beta}$ is given by the minimum $\min \left(\mu_{+}^{\alpha}, \mu_{+}^{\beta}\right)$. Conversely, any $\alpha \cup \beta$ metastable state with upper limit $\mu_{+}^{\alpha \cup \beta}$ may be obtained by combination of an $\alpha$ and a $\beta$ metastable state verifying either

$$
\mu_{+}^{\alpha}=\mu_{+}^{\alpha \cup \beta} \text { and } \mu_{+}^{\beta} \geq \mu_{+}^{\alpha \cup \beta}
$$

or

$$
\mu_{+}^{\alpha} \geq \mu_{+}^{\alpha \cup \beta} \text { and } \mu_{+}^{\beta}=\mu_{+}^{\alpha \cup \beta}
$$

A standard probability analysis leads to:

$$
\begin{aligned}
\mathcal{N}_{N}^{\alpha \cup \beta}\left(\mu_{+}\right) & =\mathcal{N}_{N}^{\alpha}\left(\mu_{+}\right) \int_{\mu_{+}}^{+\infty} \mathcal{N}_{N}^{\beta}\left(\mu_{+}^{\prime}\right) d \mu_{+}^{\prime} \\
& +\mathcal{N}_{N}^{\beta}\left(\mu_{+}\right) \int_{\mu_{+}}^{+\infty} \mathcal{N}_{N}^{\alpha}\left(\mu_{+}^{\prime}\right) d \mu_{+}^{\prime}
\end{aligned}
$$

where the first and second terms emerge from Eq. A.1 and $\mathrm{A} .2$ respectively.

We introduce the number of metastable states with a stability range larger than a given $\mu_{+}$:

$$
\mathcal{Q}_{N}^{\alpha}\left(\mu_{+}\right)=\int_{\mu_{+}}^{+\infty} \mathcal{N}_{N}^{\alpha}\left(\mu_{+}^{\prime}\right) d \mu_{+}^{\prime}
$$

Combination of Eq. A.3 and A.4 leads to a differential equation showing that $\mathcal{Q}_{N}^{\alpha}\left(\mu_{+}\right)$is multiplicative:

$$
\mathcal{Q}_{N}^{\alpha \cup \beta}\left(\mu_{+}\right)=\mathcal{Q}_{N}^{\alpha}\left(\mu_{+}\right) \times \mathcal{Q}_{N}^{\beta}\left(\mu_{+}\right)
$$

Relaxing the constraint on the lower stability limit $\mu_{-}$ produces similar calculations with extra terms leading to Eq. 3 and 4.
[1] G. Bertotti, Hysteresis in Magnetism (Academic Press, San Diego, 1998).

[2] E. Kierlik, P. A. Monson, M. L. Rosinberg, L. Sarkisov, and G. Tarjus, Phys. Rev. Lett. 87, 055701 (2001).

[3] E. Kierlik, P. A. Monson, M. L. Rosinberg, and G. Tarjus, J. Phys.: Condens. Matter 14, 9295 (2002).

[4] E. Kierlik, M. L. Rosinberg, G. Tarjus, and P. Viot, Phys. Chem. Chem. Phys. 3, 1201 (2001).

[5] J. Puibasset, J. Chem. Phys. 133, 104701 (2010).

[6] F. Detcheverry, E. Kierlik, M. L. Rosinberg, and G. Tarjus, Phys. Rev. E 68, 061504 (2003).

[7] F. Detcheverry, E. Kierlik, M. L. Rosinberg, and G. Tarjus, Langmuir 20, 8006 (2004).

[8] F. Detcheverry, E. Kierlik, M. L. Rosinberg, and G. Tarjus, Phys. Rev. E 72, 051506 (2005).

[9] D. H. Everett, Trans. Faraday Soc. 50, 1077 (1954).

[10] D. H. Everett and F. W. Smith, Trans. Faraday Soc. 50, 187 (1954).

[11] D. H. Everett and W. I. Whitton, Trans. Faraday Soc. 48, 749 (1952).

[12] S. J. Gregg and K. S. W. Sing, Adsorption, Surface Area and Porosimetry (Academic Press, New York, 1982).

[13] F. Detcheverry, M. L. Rosinberg, and G. Tarjus, Eur. Phys. J. B 44, 327 (2005).

[14] X. Illa, M.-L. Rosinberg, and E. Vives, Phys. Rev. B 74, 224403 (2006).
[15] A. Lefèvre and D. S. Dean, Eur. Phys. J. B 21, 121 (2001).

[16] M. L. Rosinberg and T. Munakata, Phys. Rev. B 79, 174207 (2009).

[17] J. P. Sethna, K. Dahmen, S. Kartha, J. A. Krumhansl, B. W. Roberts, and J. D. Shore, Phys. Rev. Lett. 70, 3347 (1993).

[18] E. Kierlik, J. Puibasset, and G. Tarjus, J. Phys.: Condens. Matter 21, 155102 (2009).

[19] F. J. Pérez-Reche, M. L. Rosinberg, and G. Tarjus, Phys. Rev. B 77, 064422 (2008).

[20] J. Puibasset, J. Chem. Phys. 127, 154701 (2007).

[21] J. Puibasset, Langmuir 25, 903 (2009).

[22] J. S. Beck, J. C. Vartuli, W. J. Roth, M. E. Leonowicz, C. T. Kresge, K. D. Schmitt, C. T.-W. Chu, D. H. Olson, E. W. Sheppard, S. B. McCullen, et al., J. Am. Chem. Soc. 114, 10834 (1992).

[23] C. T. Kresge, M. E. Leonowicz, W. J. Roth, J. C. Vartuli, and J. S. Beck, Nature 359, 710 (1992).

[24] A. Uhlir, Bell Syst. Tech. 35, 333 (1956).

[25] L. Bruschi and G. Mistura, J. Low Temp. Phys. 157, 206 (2009).

[26] L. Bruschi, G. Fois, G. Mistura, K. Sklarek, R. Hillebrand, M. Steinhart, and U. Gösele, Langmuir 24, 10936 (2008).

[27] L. Bruschi, G. Mistura, L. Liu, W. Lee, U. Gösele, and 
B. Coasne, Langmuir 26, 11894 (2010).

[28] J. Puibasset, J. Phys.: Condens. Matter 23, 035106 (2011).

[29] A. Grosman and C. Ortega, Langmuir 24, 3977 (2008).

[30] A. Grosman and C. Ortega, Phys. Rev. B 78, 085433 (2008).

[31] A. Grosman and C. Ortega, Langmuir 25, 8083 (2009).
[32] It is a highly debated issue whether the hysteresis observed in these materials arises from physico-chemical heterogeneities or elastic strain [29-31].

[33] Note that in the thermodynamic limit, to calculate the leading term of $\ln \mathcal{Q}_{N}^{\alpha}\left(\mu_{-}, \mu_{+}\right)$one may replace in Eq. 3 the integrals by a maximum and $\mathcal{N}_{N}^{\alpha}$ by $\Sigma_{N}^{\alpha}$. 